Itinéraires Itinéraires

Littérature, textes, cultures

2009-2 | 2009

Caraïbe et océan Indien

\title{
Les « déportés » de la Creuse : le dévoilement d'une histoire oubliée
}

\section{Valérie Magdelaine-Andrianjafitrimo}

\section{OpenEdition}

\section{Journals}

Édition électronique

URL : http://journals.openedition.org/itineraires/254

DOI : 10.4000/itineraires.254

ISSN : 2427-920X

Éditeur

Pléiade

\section{Édition imprimée}

Date de publication : 1 juillet 2009

Pagination : 47-64

ISBN : 978-2-296-09639-4

ISSN : 2100-1340

\section{Référence électronique}

Valérie Magdelaine-Andrianjafitrimo, «Les « déportés » de la Creuse : le dévoilement d'une histoire oubliée », Itinéraires [En ligne], 2009-2 | 2009, mis en ligne le 02 juin 2014, consulté le 30 avril 2019. URL : http://journals.openedition.org/itineraires/254 ; DOI : 10.4000/itineraires.254

Ce document a été généré automatiquement le 30 avril 2019

\section{cc) (†) $\ominus$}

Itinéraires est mis à disposition selon les termes de la licence Creative Commons Attribution - Pas d'Utilisation Commerciale - Pas de Modification 4.0 International. 


\title{
Les « déportés » de la Creuse : le dévoilement d'une histoire oubliée
}

\author{
Valérie Magdelaine-Andrianjafitrimo
}

L'histoire, ou plutôt le scandale, des «Enfants de la Creuse » est brutalement réapparue dans l'actualité réunionnaise dans les années 2000. Elle y a ressurgi par une double médiation : la parution de témoignages et d'articles dans la presse ${ }^{1}$ et la publication du récit de Jean-Jacques Martial, Une Enfance volée (2003), bientôt suivi d'autres récits de vie comme celui de Jean-Pierre Gosse, La Bête que j'ai été (2005)² et d'extraits de récits dans le recueil d'Élise Lemai, La Déportation des Réunionnais de la Creuse. Témoignages (2004). C'est donc dans l'espace public et médiatique que cette révélation s'est inscrite, et sa mise en récit visait à être au plus proche du réel. Presse comme récits de vie occupent des niches majeures dans l'élaboration du champ littéraire réunionnais (Magdelaine et Marimoutou, 2006). Ils lui permettent de se construire dans les écarts de la littérature légitimée et des représentations du lieu et du sujet créoles qu'elle a longtemps imposées. L'on ne s'étonne pas, dès lors, du retentissement qu'ont eu ces témoignages qui, de plus, renvoyaient à une période particulièrement difficile dans la vie de l'île $e^{3}$. On y retrouve un paradigme récurrent de l'imaginaire réunionnais, celui du petit Créole, dépossédé de tout, et surtout de lui-même, par des puissances hégémoniques qui perpétuent les formes de l'oppression coloniale $^{4}$. La victimisation, le pathos, mais aussi la capacité de survie du petit Créole, sont autant de ces traits redondants que les récits des enfants de la Creuse viennent réactiver et radicaliser. Par leur désir de reconstruction identitaire et de réconciliation avec leur terre natale, ils trouvent un important écho dans une île en pleine quête d'ellemême. Publiés en France, ces textes s'agrègent au récit collectif que tisse l'ensemble des chroniques et récits de vie publiés dans l'île. De plus, ils bénéficient de la caution symbolique d'une publication faite « dehors ».

2 L'histoire a ainsi progressivement été révélée. Après ces témoignages, elle a fait l'objet d'ouvrages scientifiques. Puis la fiction à son tour s'en est emparée, avec le téléfilm de Francis Girod, Le Pays des enfants perdus (France 3,2003), le récit pour la jeunesse de Brigitte Peskine, L'̂̂le de mon père (2005), le roman de la journaliste française Mariella Righini, Bonbon piment (2004), et surtout, celui du Réunionnais Jean Louis Robert, Creuse, ta 
tombe (2006). Cette mécanique du dévoilement montre le rôle fondateur du récit donné pour vrai dans la reconquête de l'histoire qui finalement ne devient objet de préoccupation qu'une fois que l'événement a été rapporté, mis en discours.

Rappelons les faits tels qu'Ivan Jabonkla les retrace ${ }^{5}$. À partir de 1963 et jusqu'en 1982, la DDASS (direction départementale des affaires sanitaires et sociales) de La Réunion ${ }^{6}$, à l'instigation de Michel Debré, préfet tout-puissant de l'île, a transféré en métropole mille six cents enfants dont certains étaient abandonnés et vivaient en foyer ${ }^{7}$. Mais le scandale concerne surtout ceux qui ont été pris à leurs familles alors que celles-ci n'avaient donné leur accord qu'avec réticence, voire avaient été dupées. Cette situation est différemment perçue par les enfants qui oscillent entre ressentiment envers des parents qui les ont trahis ou pitié pour des familles abusées. On assurait à ces familles en grande difficulté sociale, souvent illettrées, et impressionnées par les visites insistantes des services sociaux, que leur enfant serait éduqué. On leur garantissait sa réussite sociale dans le pays de cocagne que représentait pour beaucoup la « métropole». On leur disait aussi que les enfants reviendraient tous les ans dans l'île et ne perdraient pas contact avec leurs familles, ce qui ne fut pas le cas. Le choix des enfants s'est fait sur signalement d'assistantes sociales souvent formées dans l'idée que la misère était en soi une rupture $\mathrm{du}$ lien familial et qui ne faisaient pas non plus cas de la situation des enfants "ramassés ${ }^{8}$, les considérant comme abandonnés. Cette opération a été justifiée par l'angoisse de Michel Debré devant l'explosion démographique qui selon lui menaçait l'île et par le délabrement sanitaire et social de La Réunion, plus encore que par le souci d'insuffler une nouvelle jeunesse à des départements français en pleine désertification. Mais elle a surtout été rendue possible par deux paramètres que rappelle Ivan Jablonka. Tout d'abord, elle relève d'une conception de la petite enfance qui a longtemps prévalu en France : les pupilles étaient considérés comme réadaptables sans condition et devaient pour cela voir toutes les relations rompues avec leur famille et leur milieu. Elle s'inscrit surtout dans une idéologie plus vaste: le désir d'intégrer La Réunion à l'ensemble national, l'idéal républicain supputant que tout Français pouvait être transplanté sans difficulté n'importe où dans le territoire national. L'opération a été un échec: dépressions, suicides, alcoolisme, échec scolaire et social massif, ont été autant de réponses à cette politique aveugle de la part d'individus victimes de solitude et de racisme. La Creuse n'a pas été la seule région de destination des enfants, elle a même en réalité accueilli moins de pupilles que d'autres départements comme la Lozère, le Tarn ou le Gers. Mais c'est elle que l'on retient surtout, d'une part, parce que le foyer de Guéret s'est tristement inscrit dans les mémoires et, d'autre part, parce que la Creuse constituait le lieu le plus difficile à supporter pour les enfants. Il était le plus froid ${ }^{9}$, le plus isolé, celui où ils ont eu le plus à souffrir de l'exploitation dans les fermes où certains garçons ont été maltraités. Faut-il pour autant, comme le font les anciennes victimes qui ont intenté en vain un procès à l'État ${ }^{10}, \mathrm{y}$ voir une nouvelle forme d'esclavage, un système illégal de migration? Selon l'historien, «[1]a migration enfantine est donc la traduction humaine de la départementalisation; elle illustre les ravages que la loi républicaine a provoqués en déferlant sur l'ancienne colonie. Son caractère destructeur va donc de pair avec sa légalité : le scandale, en éclatant, masquera ce paradoxe » (Jablonka, 2007 : 194).

C'est en effet la part la plus scandaleuse qui en est conservée. Le surgissement de cette histoire à l'époque contemporaine et sa requalification en déportation, en esclavage, le montrent. D'une part, ils s'inscrivent dans un discours nouveau sur l'esclavage moderne. D'autre part, ils s'articulent à l'exigence actuelle de réparation de l'histoire et 
d'assignation des responsabilités qui coïncide avec l'émergence de voix subalternes dressées contre les systèmes de domination. Dans le contexte réunionnais enfin, ce surgissement d'une histoire occultée est doté d'un fort poids symbolique. La Réunion, comme les autres espaces sucriers qui ont vécu l'esclavage, l'engagisme, la colonisation puis une départementalisation aux échos coloniaux évidents, connaît une histoire violente, faite des démembrements des divers peuples qui l'ont fondée. Le discours réunionnais se caractérise par ses amnésies, par les fantômes qui le hantent. Les enfants de la Creuse, eux aussi, ont failli disparaitre de cette histoire. Ils l'ont réinvestie en s'adossant sur ce qui pour eux n'est pas une métaphore mais bien une réalité historique, l'esclavage, la déportation. Nous le verrons en analysant les récits de Jean-Jacques Martial et Jean-Pierre Gosse et le roman de Jean Louis Robert. Le fait de s'appuyer sur cette double tragédie vise à repriser les lambeaux d'une mémoire discontinue ${ }^{11}$. Ainsi mis en perspective, les événements entrent en résonance pour reconstruire une histoire postcoloniale faite de la permanence de l'exploitation des Créoles. Dès lors, il s'agit bien d'une « bataille mémorielle » qui se fait jour. Le récit constitue un enjeu majeur puisqu'il permet de convertir un manquement administratif en épisode de l'histoire coloniale. Il offre également l'opportunité à ceux qui n'étaient alors que des statistiques et des dossiers de la DDASS, de devenir des sujets.

Cette bataille mémorielle est fondamentale : alors que les pupilles ont toujours été traités en termes administratifs, comme des catégories ou comme des variables entrant dans la composition des " contingents ", ils tentent, à partir de 2002, d'être reconnus dans leur statut de victimes - comme les Noirs réduits en esclavage, les Juifs assassinés, etc. Or, désormais, ce statut est délivré autant par les médias que par les tribunaux. (Ibid. : 222)

On peut rajouter que ce statut est également délivré par le récit, qu'il soit fictionnel ou factuel. Car le récit, comme le roman, laisse entendre une voix à la première personne qui partage l'expérience de sa subalternité enfin passée à la représentation. Mais saturée de références, tant intertextuelles qu'historiques qui la fictionnalisent, la voix des enfants de la Creuse n'est-elle pas une nouvelle fois occultée, voire confisquée ? Si la comparaison avec d'autres épisodes de l'histoire constitue un travail mémoriel indispensable (Todorov, 1998 : 33-42), l'écrasement et la contiguïté des références ainsi établie ne bloquent-ils pas le travail de construction d'une mémoire exemplaire au profit d'une mémoire littérale ?

\section{Interactions fictionnelles}

6 Enfance volée, violée, exploitée, vie gâchée, suicides, maladies, dépression, alcoolisme, échecs sociaux et affectifs sont les éléments redondants de ce scandale des enfants arrachés à leur île. Le désir de récupérer sa parole est obsessionnel : on le voit chez Gosse qui dit de sa parole qu'elle a été mise «à la poubelle (Gosse, $2005: 33$ ) et qui exprime son désir de témoigner, selon le titre de l'un de ses chapitres, «La parole retrouvée » (Ibid. : 67). Il évoque son histoire « encore murée en [lui]», dans la " grande amnésie » (Ibid. : 64) collective de l'île et clôt ainsi son témoignage: "Je dédie ce récit à tous ceux qui souffrent. Puissent-ils un jour avoir la force et les moyens de rendre public leur sort afin qu'il ne soit pas nié comme l'a été trop longtemps le mien » (Ibid.: 84). Martial, à la suite de sa thérapie, se munit de cahiers et de stylos pour découvrir la fonction réparatrice de l'écriture : «J'y suis arrivé. J'ai réussi à parler et à écrire. J'ai déverrouillé ces maudites portes blindées » (Martial, 2003 : 105). Le roman de Robert, dans son préambule, évoque la naissance du désir d'écrire : 
Il préfère prendre le cahier. Les doigts droits le tiennent, pendant que le majeur glisse le long du ressort, conquérant, suivi bientôt de la ligne de vie, donnant naissance à un diguediguement agréable dans toute la main, $\mathrm{y}$ stimulant le centre de l'écriture. (Robert, $2006: 5$ )

7 Il revient à de nombreuses reprises sur la nécessité impérieuse de dire :

Mais ça déborde de toi. Tu ne parviens plus à contenir les mots en crue qui se pressent contre le mur invisible qui te sépare d'eux. Mur que tu veux briser pour retrouver un peu de ta qualité d'homme. Les mots que tu retiens te font mal. Si mal. (Ibid. : 122)

Enfin née, cette parole doit trouver le moyen de se construire. C'est en empruntant à certaines formes fictionnelles qu'elle y parvient. Seul Jean Louis Robert exploite une intertextualité savante, celle de l'épopée méditerranéenne de l'exil, avec L'Odyssée et surtout, dans l'Énéide, avec le personnage de Créuse, qui lui permet de jouer sur une déclinaison du mot Creuse.

Les autres références renvoient à des genres plus populaires, dont le conte. Le petit héros abandonné et séparé de sa fratrie évoque le Petit Poucet victime de l'ogre, ce que Jablonka relève également dans l'ensemble du discours médiatique (Jablonka, 2007 : 219). Cet Ogre prend la figure de Michel Debré, que Jean Louis Robert surnomme le Grand Démograficomaniaque, en raison de son obsession pour le contrôle de la démographie. Mais c'est surtout le choix d'une métonymie obsédante qui revient comme un profond marqueur de l'imaginaire réunionnais, et en même temps, comme un attribut maléfique de l'opposant : la $2 \mathrm{CV}$ fourgonnette qui sillonne les routes et les quartiers pauvres de l'île pour enlever les enfants. Seul Jean Louis Robert choisit d'évoquer également les assistantes sociales, renommées les "deux thaumaturgettes" en raison des miracles qu'elles promettent aux parents en prédisant un avenir glorieux aux enfants qu'elles veulent enlever. La forme du conte est explicitement utilisée chez Jean Louis Robert : le même récit revient dans deux chapitres différents du roman, 4 et 37. L'un est en français, l'autre en créole, mais tous deux sont introduits par la formule traditionnelle du conte « kriké kraké » et en reprennent la formule de clôture : «E si zistwar la lé mantèr, la pa mwin lotèr ${ }^{12} »$ (Robert, 2006 : 137).

Un autre intertexte, implicite cette fois, et qui lui-même s'appuie sur certains motifs des contes, est convoqué par ces récits : les romans de l'enfance malheureuse. Comme dans The Adventures of Olivier Twist (1837) de Dickens, les enfants, enlevés, abandonnés ou privés de leurs parents, connaissent la violence des foyers, la brutalité d'un système d'éducation inepte, les tentatives de perversion d'une société qui marginalise les plus faibles et les condamne à la déchéance, ici sous la forme de l'exploitation sexuelle, du viol, de l'alcoolisme, du sous-emploi... Chez Jean-Pierre Gosse, la description du foyer d'HellBourg est caractéristique de cette évocation :

[La piscine] a été construite par des enfants qui ont sué sang et eau. On travaillait toute la journée comme cela. On nous testait. On regardait si on était aptes. Sans doute pour la suite de ce que je pense être un programme préétabli. Le premier repas, on ne le prenait qu'à $6 \mathrm{~h} 30$. Il était constitué d'une toute petite plaquette de beurre rance et de pain rassis d'où sortaient des cancrelats. [...] Pour tout repos, on m'a fait mettre à genoux durant une demi-journée. Mais cela ne suffisait pas. Sur la tête, on m'a posé une grosse pierre que je devais tenir pour ne pas qu'elle tombe. Pour corser le tout parce qu'un gamin qui subit un tel traitement n'est pas encore assez gâté, on m'a glissé un tronc d'arbre sous les genoux. [...] Je n'ai jamais remis les pieds à la maison. Je suis resté parmi les forçats. (Gosse, $2005: 20$ ) 
11 Il ne s'agit bien sûr pas de nier la réalité historique qui préside à ces évocations puisqu'en effet, le foyer d'Hell-Bourg a une sinistre réputation bien établie. Mais on relève aisément la construction de l'effet de lecture induit par le pathos, qui garantit la reconnaissance immédiate d'une topique de la martyrologie de l'enfant. Elle est structurée selon les étapes clés que sont les châtiments corporels, la faim, la déshumanisation des figures d'adultes incarnant le mal absolu ainsi que, sur un plan socio-économique, l'exploitation $\mathrm{du}$ patronat. On les retrouve chez le père adoptif violeur de Martial, ou chez les paysans qui emploient Gosse, et font de lui un enfant sauvage : « Pour seuls amis, j'avais les porcs. J'avais fini par me déplacer comme eux quand j'étais seul : à quatre pattes " (Ibid. : 43). Cette focalisation sur les épisodes les plus dramatiques s'appuie sur des procédés d'emphase, des hypotyposes qui rendent la victimisation exemplaire. Chez Martial, l'intensification est produite par des enchaînements très oralisés qui s'adressent directement aux destinataires: "Puis vinrent de nouvelles souffrances. Il va falloir, chères lectrices, chers lecteurs, que vous vous accrochiez, que vous serriez vos mâchoires, vos poings. Mon univers allait basculer à nouveau » (Martial, 2003 : 43).

La distorsion fictionnelle participe pleinement de la recherche du pathos et de la connivence. Elle ne peut être évitée du fait même de la nature du récit mémoriel, toujours friable. Daniel Bertaux (2001) a montré comment il subit nécessairement la fictionnalisation de la sélection, de l'amplification et du passage à une narration exemplaire. D'autre part, la reconstruction permet de radicaliser les espaces, les postures, les temps, les perceptions. La Réunion fait ainsi l'objet d'opacifications dues à un désir de la transformer en espace mythique de l'enfance, ce qui réhabilite tout à la fois la terre natale et la mère biologique, toujours inscrite dans le tableau de ce bonheur primordial. Chez Martial, les champs de cannes deviennent le lieu rêvé des jeux de l'enfance, alors qu'ils incarnent, dans l'imaginaire réunionnais, la mémoire de l'esclavage, de la souffrance physique et de l'exploitation économique. Chez Gosse, l'île est reconstruite par le prisme d'une nostalgie inconsolable : la nuit s'y coucherait à 23 heures et les enfants se baigneraient en compagnie des dauphins à la nuit tombante dans les eaux de Saint-Denis, connues pour leur dangerosité13.

13 La Réunion n'apparaît comme une marâtre, empêtrée dans ses lâchetés et ses contradictions, que chez Jean Louis Robert qui y vit et qui n'a pas été « déporté ».

J'en veux à cette île-le-monde, je vais lui faire payer son indifférence à mon égard, je vais envoyer un avion percuter sa pyramide à l'envers ${ }^{14}$, pour la remettre à l'endroit, remettre l'île à l'endroit, elle a suffisamment fait le poirier comme ça, le sang des esclaves engagés déportés a suffisamment irrigué son cerveau, il faut qu'elle pense par elle-même, qu'elle comprenne que son salut ne peut venir de l'extérieur, qu'elle comprenne qu'elle n'a pas à fermer les yeux quand on déporte dé-por-te ses enfants, qu'elle comprenne qu'elle est aussi coupable que le Grand Démograficomaniaque. (Robert, $2006: 125)$

14 Ces distorsions permettent de restaurer l'ampleur du scandale. En s'appuyant sur des structures dramatiques préexistantes, les textes entraînent l'adhésion des destinataires. En même temps, ils manifestent ainsi leur difficulté à donner corps à ce qui n'en avait pas, à mettre des mots sur ce qui en était privé. Or, le mot qui est choisi est l'un des plus lourds de sens historique : la déportation, qui s'articule ici sur l'histoire de l'esclavage. 


\section{Déportation et esclavage : mémoires littérale et exemplaire}

Il ne nous appartient pas d'interroger la pertinence de ces termes, car c'est un débat qui concerne les historiens. En revanche, ils renvoient à un discours et à une formation d'identifications identitaires déjà utilisés dans d'autres contextes. C'est le sens de l'ouvrage de Paul Gilroy, L'Atlantique noir (2003), qui montre comment «l'hypo-histoire » juive, tant biblique de l'exode, que contemporaine de la Shoah, permet de structurer le discours contemporain sur l'esclavage noir souvent considéré comme récalcitrant à la mise en mots et en images. Dans le cas qui nous occupe, il s'agit donc d'un double détour. Pour dire l'histoire contemporaine des enfants, on renvoie à l'esclavage, qui est une période que l'île parvient encore difficilement à verbaliser pleinement. Et pour ce faire, on passe par la médiation d'un système de références qui relève d'une autre histoire (Magdelaine, 2008).

16 Cette modalité de la représentation de l'expérience des enfants est donc très ambiguë. Inscrite dans cette double tragédie historique, elle se voit dotée d'une puissance accrue et se trouve réhabilitée. Mais elle risque aussi de procéder malgré elle à un nouvel étouffement des voix, à une confiscation de la réalité en étendant « les conséquences du traumatisme initial à tous les moments de l'existence» (Todorov, $1998: 30$ ). Cette forme d'écriture contribuerait alors «à soumettre le présent au passé» (Ibid. : 31). L'obsession d'acquérir le privilège symbolique du statut de victime (Ibid. : 57) semble le corroborer.

La double appellation de déportation et d'esclavage est illustrée par la redondance des termes et par les faits que relatent les récits : chaînes, absence de nourriture, exploitation du corps...

Ce soir-là, je me suis senti plus esclave que jamais. Pour éviter que je prenne à nouveau le large, G. m'a attaché aux chevilles avec une chaîne. Pour débuter ma journée de travail, j'attendais comme un animal qu'on me détache. Un vrai bœuf. (Gosse, $2005: 42$ )

18 Le souci de justification du terme est constant et se caractérise par une sorte de préoccupation définitoire que l'on trouve chez Gosse :

jamais je n'ai reçu un centime pour ma peine. [...] Voilà pourquoi, en plus de ma situation physique et du traitement que j'ai dû subir, je parle d'esclavage. Effectuer contraint et forcé un labeur que l'on n'a aucune envie de mener, en souffrir, et n'être pas payé. Je ne pense pas pouvoir appeler cela autrement qu'Esclavage. (Ibid. : 40)

C'est la définition de la déportation que l'on retrouve chez Jean Louis Robert. Le narrateur renvoie à l'autorité du dictionnaire :

Le dictionnaire dit qu'il s'agit d'un ex-île dans un lieu déterminé et c'est bien d'une sortie de l'île dont il est question. Il s'agit aussi, d'après le dictionnaire, d'un internement dans un camp situé à l'étranger ou au-delà des mers. Nous avons bien dessauté l'Océan Indien pour atterrir dans des camps en France, dans le Gers, dans la Creuse ou ailleurs. Vous voyez, la vérité est dans le dico. Monsieur le Démograficomaniaque, nous avons bien été déportés, et nous ne vous saluons pas. (Robert, 2006 : 143)

20 Aucun autre mot n'est plus possible : l'écriture vise à résoudre sans coup férir le débat historique. Certains titres ou sous-titres le fixent dans la conscience du destinataire : « La Bête que j'ai été. Le témoignage d'un Réunionnais déporté dans la Creuse en 1966 » pour 
Gosse, «La Déportation des Réunionnais de la Creuse. Témoignages » pour le recueil d'Élise Lemai (2004). Le collage de l'idée de déportation et de témoignage rend toute contestation impossible.

21 C'est aussi l'objectif que visent les documents d'escorte. Fac-similés, photos, documents officiels alimentent le scandale. La légende des photos chez Martial le souligne. Le doigt dont on voit l'empreinte sur son acte d'abandon ne peut être celui de son père : « Ce doigt paraît bien fin pour un coupeur de cannes à sucre » (Martial, 2003 : 110). L'administration a bien menti, ce qui entérine le vol et la déportation de l'enfant. L'échange de courrier entre le professeur Denoix qui pour la première fois a utilisé le terme de déportation et un Debré caustique est également reproduit. Chez Jean Louis Robert, cette célèbre lettre de Debré est réécrite. Quelques pans (soulignés ici) en sont conservés. L'ironie du député, inscrite dans celle du narrateur, rend ses propos insoutenables lorsqu'on les confronte à la réalité de la vie des enfants : « Mes enfants, prunelles de mes yeux, je souhaiterais qu'il existât de nos jours un nouveau Molière apte à dépeindre ce groupe de psychiatrie infantojuvénile Mes enfants, pourquoi ne m'acclamez-vous pas? Où êtes-vous?» (Robert, 2006 : 127).

La construction du roman de Jean Louis Robert accentue l'écho entre ces épisodes historiques au point de les rendre indémêlables. Les jeux de l'onomastique permettent de dédoubler les personnages et leurs itinéraires. Leur identité est fluctuante entre marrons, esclaves et enfants. Le lecteur, égaré entre les niveaux de lecture et d'interprétation, les confond et mêle les temporalités. L'enfant de la Creuse rêve d'une interpénétration des histoires dans une sorte de résurgence mémorielle involontaire qui garantit une filiation directe entre les temporalités :

Nuit après nuit, les mêmes éléments revenaient: fouet, fleur de lys; oreilles coupées ; poule noire ; carcan ; chaînes ; pendaisons ; beaucoup de poules noires ; volcan ; jarrets coupés; mains clouées à des troncs d'arbres flamboyants; volcan en éruption ; autos rouges ; $2 \mathrm{CV}$ camionnette grise ; sabres à cannes ; neige recouvrant des cratères incandescents ; incendies... Cette nuit-là, dans mon rêve, je participais à un jeu de rôles avec un camarade. Au grand jeu de l'Abolition. (Ibid. : 67)

L'aliénation postcoloniale subie par les enfants s'inscrit dans le suivi des exploitations coloniales qui ont terrassé l'île et ne l'ont pas encore pleinement libérée. La tragédie devient une fatalité pour La Réunion. En même temps, elle permet d'affronter une question cruciale dans une île oublieuse, voire amnésique, la question de la mémoire.

Jean Louis Robert analyse les interactions entre un trop-plein de mémoire et une tentation de l'oubli qui rongent lîle.

Untel, souffrant d'excroissances tumémorielles qu'il s'efforçait de réduire en recherchant à tout moment le trou de mémoire et qui était sujet à de très violentes réactions si on avait le malheur de prononcer ou s'il lisait certains mots... retour du trop... le trou s'emplit de la Creuse qui gonfle, s'amplifie, stalagmite en étron. L'excès de merde déborde imprégnant les moindres interstices de la souvenance... La Creuse... Creuse... toujours... jusqu'à la fin de ta vie... jusqu'à ce qu'on creuse ta tombe. (Ibid. : 145)

Il laisse ainsi apparaître la fonction du récit : il agit comme un électrochoc, qui éveille, par la puissance des mots et du réel qu'ils dévoilent, une mémoire toujours latente.

La relecture de cet épisode historique à la lumière de ce double paradigme de la déportation et de l'esclavage permet donc d'explorer la question de ce que Ricœur nomme l'injonction mémorielle contemporaine. L'écriture de ces récits apparaît comme indispensable pour mettre au jour un refoulé de plus de l'histoire réunionnaise. Le faire 
sous les auspices de ce double événement traumatique dont l'un relève du passé de l'île, et l'autre pas ${ }^{15}$, traduit la difficulté à résoudre les interactions de ce que Todorov nomme mémoire littérale et mémoire exemplaire. Le refus de faire de l'esclavage et de la déportation des métaphores, mais la revendication d'une contiguïté et d'une similitude des expériences inscrit les récits dans la mémoire littérale, indépassable, d'un traumatisme dont le deuil ne peut se faire. C'est ce qui construit une victimisation répétée ad libitum, dont la dimension régressive est évidente puisque l'on parle toujours des « enfants ». Ce statut d'enfant ne s'efface pas: la souffrance n'est jamais apprivoisée, ce qui bloque la possibilité d'un présent d'adulte. La quête de la mère et d'une réconciliation avec elle contribue à cette infantilisation de l'éternelle victime, de l'Ogre, des puissants, des adultes, de la France. Le récit de Gosse paraît bien inscrit dans cette mémoire, d'autant que l'état de souffrance du narrateur est tel qu'il lui est difficile de se reconstruire. En revanche, Martial semble élaborer progressivement une mémoire exemplaire lorsqu'il traine la France en justice, propose la création d'associations de Réunionnais. Mais le dépassement se fait dans le réel plus que dans le récit. Après avoir lancé une déclaration de guerre contre l'esclavage moderne, son texte se clôt sur une injonction à rapatrier les déportés de la Creuse vivant encore dans des foyers en France, et à rendre leur terre à ceux qui sont morts et enterrés loin de chez eux. La tentation régressive, la «nostalgie du retour au lieu le plus archaïque du commencement absolu » (Derrida, 1995 : 142), l'indépassable du passé sont donc encore bien présents. La mémoire exemplaire, celle qui permet de tirer une leçon du passé, de l'utiliser « en vue du présent, de se servir des leçons des injustices subies pour combattre celles qui ont cours aujourd'hui, de quitter le soi pour aller vers l'autre» (Todorov, 1998:32-33) n'est que partiellement exprimée ici, car ce n'est pas vers l'autre que va le narrateur-auteur, c'est vers le même.

Il n'est pas question ici d'évaluer le bien-fondé d'une mémoire par rapport à l'autre, mais d'en voir les implications dans la fonction des récits et dans l'élaboration du discours réunionnais. La difficulté de ce que Todorov appelle un «bon usage » de la mémoire y est évidente. En cela, la réflexion insulaire s'inscrit dans la préoccupation internationale du désir mémoriel, de l'obsession commémorative qu'analyse Régine Robin (2003). Il s'agit plus spécifiquement ici, par ce «nouveau culte de la mémoire » de construire une identité collective enfin revisitée: "c'est en se constituant un passé commun qu'on pourra bénéficier de la reconnaissance due au groupe. Le recours au passé est particulièrement utile lorsque les appartenances sont revendiquées pour la première fois » (Todorov, 1998 : 53-54). Et c'est bien de cette intensité de la première fois dont relèvent nos récits. Ils permettent la construction d'un discours collectif, d'un retour commun sur un passé traumatisant. Par l'empathie qu'ils provoquent, ils ramènent chacun à l'individualisation de la souffrance, à un retour sur soi mais ils tendent à l'enfermement dans l'émotivité et le pathos.

Or, par rapport à eux, le roman rend possible le saut de la « désindividuation » (Ibid. : 32) qui permet de s'arracher à l'émotion, à la répétition obsessionnelle de l'épisode traumatique. Prenant appui sur la même mise en relation des événements tragiques de l'histoire, Jean Louis Robert les inscrit dans un ensemble de mécanismes d'oppression au premier rang desquels il place la dépossession de la langue créole. En montrant la corrélation entre la problématique de la langue et celle de l'histoire, il propose un décalage vers une mémoire exemplaire reconquise. Le passé peut s'intégrer dans le présent et poser les prémisses d'une reconstruction d'un langage et d'un rapport au 
monde réunionnais. D’ailleurs, le roman se passe presque exclusivement dans l'île, alors que les récits se passent essentiellement en France. Le décalage des préoccupations est donc évident.

\section{Reconstruction d'un langage et d'une représentation romanesques}

Creuse, ta tombe est entièrement construit sur le principe de l'écho, de la fusion, voire de la confusion. Il fonctionne comme un kaléidoscope, mimétique de la situation de perdition et d'égarement du narrateur. Ce dernier est atteint d'une maladie de l'identité ou, selon les psychiatres, d'un «trouble de la personnalité multiple» qui «se traduit par la présence de deux, ou plus, personnalités distinctes chez une même personne. Il serait la conséquence d'abus sexuels subis pendant l'enfance» (Robert, 2006: 139). Or cette fragmentation se reporte sur la structure narrative, découpée en un ensemble de chapitres qui font alterner les voix, les personnages, les tonalités, les temporalités, les lieux, les genres littéraires. Conte, dialogue théâtral, roman, litanie onomastique, listes, lettres, articles de dictionnaire, expression poétique, déchaînement lettriste avec l'alternance $O / Q$ (jouant sur le syntagme créole " $\mathrm{o} \mathrm{ki} \mathrm{»,} \mathrm{«} \mathrm{dans} \mathrm{la} \mathrm{misère} \mathrm{»)} \mathrm{sont} \mathrm{autant} \mathrm{de}$ ces formes fluctuantes qui traduisent la fluidité et l'hybridité insaisissables de l'œuvre. Toutes les possibilités du discours et de la langue sont ainsi explorées. L'expérience des enfants se disperse et s'étend dans toutes les modalités du dire, au risque même de ne plus arriver à rien dire, de menacer la construction d'un discours sur l'histoire. L'auteur joue en effet d'une distorsion immédiate de l'exigence du vrai. Il déjoue également les codes du pathos en perturbant le lecteur par une exploitation systématique, délirante, de la scatologie, du bas corporel, de la coprolalie.

30 À scandale, scandale et demi : à celui de l'histoire répond celui de l'écriture qui prend comme «fondement » le motif obsédant du « cul » au point de réduire l'individu à cette synecdoque, à cette portion congrue, et incongrue. Le «cul» et la «merde » sont les refrains de cette écriture d'un bas corporel proliférant qui déroute et dérange :

...remuant le couteau dans la plaie, la queue du dit père remue dans l'anus de l'enfant... son anus ouvert, béant... toute la merde de l'univers, qui flotte partout, tournoie de plus en plus fort, y pénètre, l'emplit complètement, se fond, se confond avec lui, il ne fait plus qu'un avec la merde de l'univers... cette merde qu'immobile il sent dans l'œil du psy... son camérœil creuse le magma de ma parole... ma parole creuse qui tient le sens au secret dans ses interstices... creuse... je me retourne comme on tire la châsse... évacuer la merde... (Ibid. : 41)

31 Le carnavalesque sans jubilation bouleverse les règles du pouvoir social mais surtout discursif et narratif. L'anus, lieu du viol, symbole de la soumission et de la victimisation de l'enfant, se fait aussi injure provocatrice, litanie puérile, comptine scandaleuse. Par un permanent déplacement métaphorique, l'organique devient magmatique, l'anus, une faille obscure et angoissante à l'image des fissures du volcan qui menacent l'ordre français. Anus et matière fécale renvoient au cratère du volcan et à la lave, et cette association éruptive annonce une destruction, paradoxalement cathartique, par la souillure :

Ils [Les Blancs] voyaient l'Édifice se craqueler... prêt à s'écrouler... se désagréger... de petites fissures se produire... tracer partout sur le bloc compact de l'Édifice de nombreux sentiers de marrons... se rejoindre, se réunir pour former une faille - La 
Réunion, une faille - s'élargir progressivement. Menacer l'ensemble. La Réunion une faille? Non. Vite obturer tout cela. (Ibid. : 17)

Le fécal et le magmatique sont à l'origine d'un lent mécanisme de création. Fabriqué à partir de l'excrémentiel, le cosmos insulaire est porteur d'une souillure inaugurale, mais en même temps, il parvient à transmuer l'ordure en principe constructif. Il laisse même augurer la découverte, au fond de la faille, de la liberté : « Ma la vi... inn ti limyèr dan la nwit... Astèr na pi ryin pou arèt amwin ${ }^{16} »($ Ibid. : 29).

Comment ne pas penser à l'omni-niant crachat de Césaire lorsque, jusque dans le principe de la litanie et de la succession sans ponctuation du discours rapporté et mis à distance par l'ironie, l'auteur semble retrouver certains échos du poète?

Elle arborait un sourire rempli de promesses Un bel avenir des études poussées des métiers pas sots du tout avocat notaire architecte fonctionnaire professeur docteur et des sucres d'orge plein de sucres d'orge. (Ibid. : 12)

4 Du cœur de cette écriture folle, proche des éructations d'Artaud, émerge la proposition de refondation d'une poétique, d'une langue et à travers elles, de l'histoire réunionnaise. Le roman glose son propre mécanisme d'élaboration: "Il faut que ça grouille, que ça foisonne, que ça dissonne mais dans la joie d'un immense et synesthésique métissage » ( Ibid. : 116).

Cette synesthésie, image des plus efficaces pour définir le processus de créolisation, permet de mettre en scène une double naissance, celle du nom propre et de la langue créole. Comme l'esclave, l'enfant a été dépossédé de son nom par la violence étatique.

Elle [mon identité] oscille d'un nom propre à un autre, nom naguère propre, le nom que je porte n'est pas mon nom, celui que j'ai porté n'est plus mon nom, quel est mon nom? Ai-je un nom? Ai-je un propre qui puisse m'ancrer ? Suis-je à jamais un ex-proprié ? (Ibid. : 124)

36 Au cœur de ces nominations possibles, il choisit de nier son « nom sale », celui qui évoque l'esclavage, Fidèle, ou le racisme auquel il est en butte, Négro, pour se réinventer un «nom propre », Tanan, tiré de ses rêves de marronnage. C'est en créole qu'il le formule : «Mon non sé Tanan. Ma la pa Fidèle » (Ibid. : 52).

La question de l'identité est en effet totalement corrélée à l'aventure de la langue. Jean Louis Robert est connu pour son désir d'« inventer une force romanesque nouvelle qui traverse les frontières génériques pour dire en mélangue l'indécidable de l'identité » (Robert, 2004: Avant-propos), comme il l'écrit dans À l'angle malang. Les maux d'ici. Le " mélangue », " mélange et appropriation des langues », "n'est pas qu'une construction formelle; il est la forme-sens qui porte et dit la complexité et l'hétérogénéité du réel réunionnais qui, en retour, le fonde " (Marimoutou, 2006: 219). Proposition de prise en charge de ce réel, il a une "dimension anthropologique» (Ibid.: 234) majeure qui lui permet d'ancrer les sujets qu'il crée dans un contexte culturel créole enfin énonçable et représentable dans toute sa complexité, manifestée par la co-présence et le dialogue des langues :

In nafèr flou qui prend naissance dans le Grand Pays Nègre, n'a pas le temps de trouver une moulure adulte, se perd en dessautant la mer au fond d'une cale, prend pied mort-né sur l'île et à partir de là, recommence à se chercher dans le foutaque de la souvenance servile, reprend forme floue dans l'élancinance du maloya, dans les accès de violence des moringueurs, s'écoule dans les katsink zistwar contées les rares jours de fête par les vieux du camp de marrons qui, après avoir frayé une tralée de sentiers, l'ont trouvée laba-an-o des mornes, là-bas au pays des morts. (Robert, 2006 : 88) 
ire des enfants est dite à la double lumière de l'histoire et de la langue de leur île natale. Ils sont ainsi réinscrits dans leur lieu et leur univers culturel, au lieu d'être laissés à l'abandon dans le vide de leur identité et de leurs origines.

Du massacre, du chaos, de l'hybridité narrative et linguistique naît une œuvre qui ne cesse de s'interroger sur elle-même, sur la langue, sur le fait d'écrire, de dire. La déclinaison permanente du mot "creuse " à travers "Creuse ", "creux ", «cul » mais aussi « créole » conjoint Creuse et créole dans le conflit et le ressentiment certes, mais elle leur redonne simultanément une place dans le même espace de la page et du livre :

c'est comme l'entame d'une question sans réponse... qui ? quoi ? quand ?... non... ça n'est pas produit par qu... ni par l'emblème de la langue d'en bas... ce $\mathrm{K}$ interdit d'écriture qui s'écrit malgré tout... plutôt comment ? ... c'est piquant au goût... ça crisse comme la langue d'en bas... le créole... cr... c'est cela... l'acre... la... cr... se précipite dans le trou en tournant dans le sens des aiguilles d'une montre... la turbulence s'accroît... appel de cr aux sons capables de colmater la béance... une tralée de faunèmes s'empressent de répondre à l'appel... casting instantané... ne font pas l'affaire... et puis viennent en rafales crépitantes tueuse menteuse voleuse vicieuse imposteuse obséquieuse... crissement de cr... se hisse hors du trou... les ampute de leurs finales... se gonflent jusqu'à ne former qu'un suffixe unique qui court s'attacher à cr... [...] La Cr... Cr... toujours... jusqu'à la fin de ta vie... jusqu'à ce qu'on... ta tombe... [...] Alors tu vomis toute la violence qu'on t'a fait subir là-bas dans la Cr. Le revoilà, le mot maudit. (Ibid. : 47)

L'aventure des enfants apparait alors comme issue d'un conflit de mots, de la victoire d'une syllabe sur l'autre. Entre Créole et Creuse, il n'a fallu que quelques lettres pour que le destin de l'enfant bascule et c'est à la langue française qu'il le doit. Mais à partir de ce rapport linguistique enfin mis au jour, peut apparaître le souci d'organiser le discours et de trouver une figure de rhétorique apte à éructer la haine de ce que l'on a subi :

Quelle figure de rhétorique suis-je ? [...] maintenant je sais la figure que je suis ? On m'a il m'a ils m'ont le père-vert et le Démograficomaniaque (épanorthose) exproprié de mon propre. Là-bas, au bout du monde, dans le trou hémorroïdal du cul du monde, en $\mathrm{Cr}$ (dégradation). Les enculés. Désancré à jamais. À jamais sans propre (chiasme). Abusé. Je suis l'Abus. La Catachrèse. CataCreuse ma tombe. Je me meurs, je suis mort, je suis enterré. (Ibid.: 38)

Exhumer la rhétorique classique pour lui faire dire l'excrémentiel, et la mettre en rapport avec la déconstruction du langage, c'est proposer un travail d'exorcisme qui jette dans le trou tout à la fois l'identité brisée et la langue coercitive qui a contribué à la défaire. Le sacrilège poétique fait naître un langage où resurgit la langue interdite. Ainsi se construit une poétique qui refonde l'histoire.

L'histoire des enfants de la Creuse est alors outrepassée, mais pas pour autant dépossédée ni censurée. Au contraire, elle est intégrée au mécanisme de la créolisation et de l'hétérogénéité du réel réunionnais. Le mécanisme de « dés-individuation » de la mémoire exemplaire lui permet de conter la trajectoire de tous les enfants sous le couvert d'un seul narrateur. Le dernier chapitre, le chapitre 54 , "Brèves ", égrène en une sorte d'oraison funèbre, la litanie de noms d'enfants aux destins tragiques rapidement résumés auxquels le narrateur a emprunté des pans de leur vie. Tous sont en lui et il les incarne tous.

Marcel Ella (ndd), fonctionnaire, qui amputa sa mère des quatre membres après l'avoir tuée, un an jour pour jour après leurs heureuses retrouvailles; Marius Véronique (ndd), mineur, qui perça son dit-père et sa dite-mère de dizaines de coups de couteau de boucher [...]. (Ibid.:144) 
43 système de signification qui, à défaut d'apaiser l'individu, laissé à sa déréliction, reconstruit l'ensemble des pans de l'histoire, de la société et de la langue réunionnaises jusque dans leurs recoins les plus purulents. Le meurtre subi ou perpétré par les enfants constitue, comme la déportation et l'esclavage, les pans d'une histoire feuilletée, créolisée qui, tout tragiques qu'ils soient, se trouvent éclairés par le travail iconoclaste de déconstruction et de recomposition de la langue qui les subsume. Là où le récit de vie contribue à faire éclater un scandale en donnant l'illusion du réel, la fiction romanesque rapièce l'histoire en inventant un nouveau langage romanesque. C'est par ce décalage qu'elle rend possible une mémoire exemplaire.

L'histoire des enfants et de leur brutale apparition dans l'espace et le débat publics pose donc la question du pouvoir et des fonctions du récit. Surgissement de voix subalternes refoulées et occultées, le récit apparaît comme un enjeu majeur dans la bataille mémorielle qui est au cœur de la construction du discours réunionnais contemporain pour définir une identité créole et la meilleure distance à adopter à l'égard de la France, la «métropole». Mais il joue aussi un rôle essentiel dans l'élaboration de la représentation, la détermination du vrai et de la fiction, ainsi que dans la légitimation des formes discursives dans le champ littéraire réunionnais. Se donnant comme au plus proche du vrai, du réel, d'un passé revenu dans un présent qui l'avait oublié, le récit de vie remplit une fonction identitaire importante dans la construction de la représentation que les Réunionnais se font d'eux-mêmes. Considéré comme savant, le roman demeure l'apanage d'une littérature canonique perçue comme allogène. Le travail de «mélangue » de Robert, largement ignoré du public réunionnais, a pourtant une valeur heuristique et contribue lui aussi à sa manière, à explorer le réel insulaire. Le récit de vie propose de construire des microhistoires empreintes de fiction qui sont autant de pièces à verser au dossier des rapports de l'île avec la France et qui comblent les blancs des archives de l'État français en donnant l'épaisseur du sujet et de l'émotion à ce qui n'était que statistiques. Pour sa part, le roman archive les négociations linguistiques à l'œuvre dans le langage créole et la complexité des mémoires qui s'y renouent.

BIBLIOGRAPHIE

\section{Récits et romans}

Gosse, Jean-Pierre, 2005, La Bête que j'ai été. Le témoignage d'un Réunionnais déporté dans la Creuse en 1966, Amélie-les-Bains, Alter Ego.

Lemai, Élise, 2004, La Déportation des Réunionnais de la Creuse. Témoignages, Paris, L'Harmattan.

Martial, Jean-Jacques, 2003, Une enfance volée, Paris, Les quatre chemins.

Peskine, Brigitte, 2005, L'île de mon père, Paris, Casterman.

Itinéraires, 2009-2 | 2009 
Righini, Mariella, 2004, Bonbon piment, Paris, Grasset.

Robert, Jean Louis, 2004, À l'angle malang. Les maux d'ici, Saint-Denis, Éditions Grand Océan, coll. « Le roman de l'Océan Indien ».

,- 2006, Creuse, ta tombe, Ille-sur-Têt, K’A.

\section{Articles et ouvrages}

Ascaride, Gilles, Spagnoli, Corine et Vitale, Philippe, 2004, Tristes Tropiques de la Creuse, Romainville, K’A.

Bertaux, Daniel, 2001, Les Récits de vie, Paris, Nathan Université, coll. « 128 ».

Derrida, Jacques, 1995, Mal d’archive, Paris, Galilée.

Gilroy, Paul, 2003 [1993], L'Atlantique noir, Paris, Kargo.

Gopal, Priyamvada, 2006, « Lire l'histoire subalterne », dans Neil Lazarus (dir.), Penser le postcolonial. Une introduction critique, Paris, Amsterdam, p. 229-258.

Jablonka, Ivan, 2007, Enfants en exil. Transfert de pupilles réunionnais en métropole (1963-1982), Paris, Seuil.

Magdelaine-Andrianjafitrimo, Valérie, 2008, «Ethnicisation ou créolisation ? Le paradigme de la Traite dans quelques romans francophones mauriciens contemporains ", dans Julia Waters (dir.), "L'ici et l'ailleurs » : Postcolonial Literatures of the Francophone Indian Ocean, [En ligne], http:// www.reading.ac.uk/web/FILES/e-france/Magdelaine.pdf.

Magdelaine-Andrianjafitrimo, Valérie et Marimoutou, Jean-Claude Carpanin, 2006, Univers créoles 6. Le Champ littéraire réunionnais en questions, Paris, Anthropos-Economica.

Marimoutou, Jean-Claude Carpanin, 2006, « Poétique du mélangue et du malang dans À l'angle malang. Les maux d'ici de Jean Louis Robert », Revue de littérature comparée, « Les littératures indiaocéaniques ", $\mathrm{n}^{\circ}$ 318, avril-juin, p. 213-234.

Robin, Régine, 2003, La Mémoire saturée, Paris, Stock.

Todorov, Tzvetan, 1998 [1995], Les Abus de la mémoire, Paris, Arléa.

Vergès, Françoise et Marimoutou, Jean-Claude Carpanin, 2005, Amarres : créolisations india-océanes, Paris, L'Harmattan.

\section{NOTES}

1. La révélation s'est faite en trois temps, dont deux qui n'ont pas «pris » dans l'esprit du public : le journal communiste réunionnais Témoignages l'a évoquée en 1968, le journal Libération et des émissions télévisées l'ont reprise en 1993, mais ce n'est qu'en 2001 que par l'entremise de Libération et du journal le JIR (Journal de l'île de La Réunion) Jean-Jacques Martial a pu apporter son témoignage et faire éclater le scandale. Voir Jablonka, $2007: 206$ sq.

2. Le texte de Jean-Jacques Martial ne relève pas à proprement parler du récit de vie puisqu'il annonce l'avoir écrit seul, alors que le témoignage de Jean-Pierre Gosse est écrit «en collaboration avec Guillaume Clavaud » qui a recueilli ses propos.

3. Dans les années 1960, La Réunion est une île du Tiers-monde où les infrastructures, les conditions sanitaires, l'habitat sont très précaires, voir Jablonka, $2007: 100$ sq. 
4. Voir Valérie Magdelaine-Andrianjafitrimo, «Le "petit" Créole et le monde. De l'ambiguïté d'un paradigme à la proposition d'une poétique de l'insularité dans trois romans réunionnais de la mémoire », Palabres, Université de Calgary (à paraître).

5. Voir aussi Ascaride, Spagnoli et Vitale, 2004. Rappelons que l'on retrouve dans de nombreux pays des situations proches, en Australie, en Acadie ou en Grande-Bretagne.

6. Historiquement, la migration des enfants est à associer à celle des adultes partis dans le cadre du BUMIDOM (Bureau pour le Développement des Migrations Intéressant les Départements d'Outre-Mer) dans les années 1960-1970 et qui seront eux aussi souvent malheureux, comme le montre le roman de Christian, Zistoir Kristian, Mes-aventures, Histoire vraie d'un ouvrier réunionnais en France, Maspero, coll. « La Mémoire du peuple », 1977, réédité chez K’A en 2009.

7. Les enfants qui vivaient en foyer à La Réunion ont souvent été heureux de partir, car seul le départ pouvait leur garantir une amélioration de leur sort. Ils semblent en général s'être mieux adaptés, mais on ne les entend pas car l'actualité est encore si tendue autour de cette redécouverte de l'histoire, qu'elle laisse peu de place à des voix discordantes qui ne stigmatisent pas ce système.

8. Les enfants qui n'ont pas de parents, ou dont les parents ne s'occupent pas, sont souvent «ramassés» par de la famille plus ou moins proche, voire par des voisins et souvent parfaitement intégrés à leur nouveau milieu, or la DDASS de La Réunion dans les années 1960 considère que « pour qu'il y ait famille, il faut père et mère » (Jablonka, 2007 : 177).

9. Ce paramètre peut sembler anecdotique mais il revient très fortement dans tous les témoignages et constitue l'une des violences majeures du choc de la métropole pour les enfants, d'autant que les premiers déplacements ont eu lieu en décembre, période la plus chaude de l'été austral, ce qui a engendré une idée d'altérité et d'hostilité du lieu très puissante.

10. Plainte rejetée par le commissaire de la République le 21 juin 2008 , décision confirmée par le Conseil d'État le 11 juillet 2008, en raison de la prescription de quatre ans qui couvre toutes les actions intentées contre la puissance publique. Voir le JIR du 12 juillet 2008.

11. Sur cette problématique, voir Vergès et Marimoutou, 2005.

12. "Si cette histoire n'est pas vraie, je n'en suis pas l'auteur."

13. On trouve des errements lexicaux également dans le texte notamment avec la définition surprenante donnée au mot «Yab», ici « yable » (Gosse, $2005: 19)$.

14. La « pyramide inversée » est le surnom de l'hôtel de Région, à Saint-Denis.

15. Le Dernier Frère de Nathacha Appanah (Paris, L'Olivier, 2007), a toutefois rappelé une autre mémoire oubliée : en 1940, 1500 Juifs, refoulés de Palestine, furent déportés à l'île Maurice, alors colonie britannique, où ils furent internés dans un camp durant toute la guerre.

16. «J'ai vu... une petite lumière dans la nuit... Maintenant il n'y a plus rien pour m'arrêter. »

\section{RÉSUMÉS}

Le scandale qui entoure les « Enfants de la Creuse », environ 1600 enfants de La Réunion envoyés dans les zones les moins peuplées de la France de 1963 à 1982 sans espoir de retour et selon les ordres de Michel Debré, préfet de La Réunion à l'époque, a explosé dans les médias en 2002 après une longue période d'amnésie collective sur le sujet. Les expériences de vie de Jean-Jacques Martial et de Jean-Pierre Gosse ont été une contribution essentielle au rappel soudain des événements. Ils ont été suivis par des romans, parmi lesquels Creuse, ta tombe de Jean Louis Robert 
qui déconstruit complètement l'histoire de La Réunion afin de créer des résonances entre les différents systèmes d'esclavage connus sur l'île. Pour raconter l'histoire des enfants, les textes se concentrent en fait sur une diversion paradigmatique double : la déportation et l'esclavage. Une fois de plus, ces deux références privent les enfants de l'unicité de leur expérience. Cependant, en même temps, elles participent à la construction d'une « mémoire exemplaire » à La Réunion.

The scandal surrounding the "Enfants de la Creuse"-approximately 1,600 children from Réunion, sent to the less populated areas in France in 1963-1982, with no hope of return, on the orders of the then prefect of Réunion Michel Debré-exploded in the media in 2002 after a long period of collective amnesia on the subject. The life stories of Jean-Jacques Martial and JeanPierre Gosse were an essential contribution to the sudden remembering of what happened. They were followed by novels, among which Jean Louis Robert's Creuse, ta tombe, which completely deconstructs the history of Réunion in order to create echoes between the different enslavement Systems known in the island. In order to tell the story of the children, the texts in fact focus on a twofold paradigmatic diversion: deportation and slavery. These two references once more deprive the children of the uniqueness of their experience. Yet at the same time they contribute to the construction of an "exemplary memory" in Réunion.

\section{INDEX}

Mots-clés : Réunionnais de la Creuse, déportation, refoulé historique, mémoire exemplaire, mémoire littérale

Keywords : Reunion Islanders in the Creuse area, deportation, historically repressed memories, exemplary memory, literal memory

\section{AUTEUR}

\section{VALÉRIE MAGDELAINE-ANDRIANJAFITRIMO}

Université de La Réunion, LCF (UMR 8143) 